\title{
Using Physiological Parameters to Evaluate Operator's Workload in Manual Controlled Rendezvous and Docking (RVD)
}

\author{
Bin $\mathrm{Wu}^{1}$, Fang $\mathrm{Hou}^{2}$, Zhi Yao ${ }^{1}$, Jianwei $\mathrm{Niu}^{2}$, and Weifen Huang ${ }^{1}$ \\ ${ }^{1}$ State Key Laboratory of Space Medicine Fundamentals and Application, China Astronaut \\ Research and Training Center, Beijing, 100094, P.R. China \\ ${ }^{2}$ Department of Logistics Engineering, University of Science and Technology \\ Beijing, Beijing, P.R. China \\ wubinaccesina.com
}

\begin{abstract}
How to train the astronauts for complicated operation in spaceflight such as manually controlled rendezvous and docking (RVD) is a common and important question. In this paper, two training methods named Routine method and Confusing method were proposed from astronaut training experience. Two-factor experiment was conducted. The factors are training method and operation difficulty level. Sixteen male subjects participated in this experiment. Eight participants were trained with Routine method and the other eight trained with Confusing method. Physiological parameters, such as respiratory rate, body temperature, heart rate (HR) and heart rate variability (HRV), were adopted as the dependent variables to evaluate operator's workload. Results show that there are significant differences on body temperature, respiratory rate, cubic root of high frequency component (HF) of HRV, normalized low frequency component $(\mathrm{LFNu})$ of HRV, and mean heart rate (HR) ( $p$ values are $0.010,0.000,0.042$, 0.009 and 0.000 , respectively) between training methods, while other physiological parameters show no significant difference. Furthermore, only significant difference on body temperature ( $p$ value is 0.010 ) was found between different operation difficulty levels. Therefore, suitable training methods deserve thorough investigation since they have significant effects on the physiological workload of the operators. In conclusion, this work provides insight into the effect of some influencing factors to workload during the RVD procedure, and will benefit the astronaut training in future.
\end{abstract}

Keywords: Rendezvous and Docking (RVD), Training method, Difficulty level, Physiological parameter, Work load

\section{Introduction}

In long-term spaceflight and deep space exploration, the spaceflight operations of the astronauts become more difficult and complicated [1]. A challenge is how to coordinate the conflict of the requirement of complex operations and limited resource [2]. This dilemma is especially prominent for final RVD phase in spaceflight. This paper aims to 
explore suitable and efficient training methods for complicated operations in final man controlled RVD phase.

Docking technology has been studied since 1960's. ETS-VII docking system developed by National Development Agency (NASDA) for unmanned spacecraft is an excellent autonomous docking system, but the system needs a high accurate onboard control system with many complicated sensors [3]. For the sake of safety and reliability, the manual RVD is more suitable in current practice for China. The Apollo spacecraft docking system is a very reliable example for manual space flight. The system did not need an onboard control system because the astronauts complete the docking procedure [3]. Other docking systems for manual space flight, such as International Space Station, Soyuz, and Mir, also adopt the similar mechanism to the Apollo docking system [3]. The object vehicle's state displayed on the screen in front of the astronauts in typical manual RVD tasks was as shown in Fig. 1. Taking Soyuz spacecraft as an example, under the aid of a television camera shooting device and an optics sighting telescope, the astronauts are able to manipulate the motion of the chasing spacecraft in $\mathrm{X}, \mathrm{Y}$ and $\mathrm{Z}$ axis by using the translation controller on the left and to control the attitude of the spacecraft by using the orientation controller on the right [4]. Since it is difficult for researchers to realize the microgravity environment on the ground, then a manual control RVD simulation system is constructed $[5,6]$. This system is based on the international engineering experience. It can simulate the scenario and provide the information that the astronauts need to accomplish the tasks.

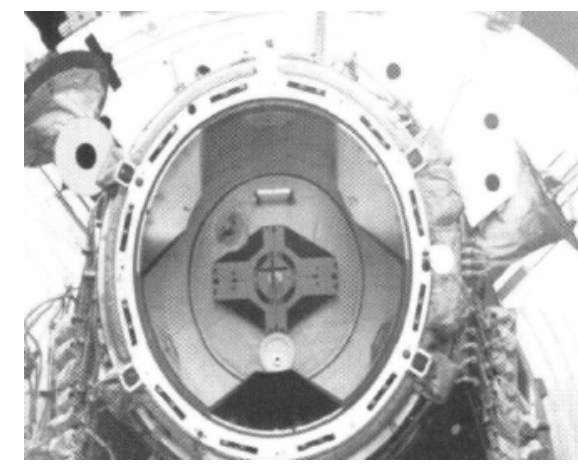

(a) USA

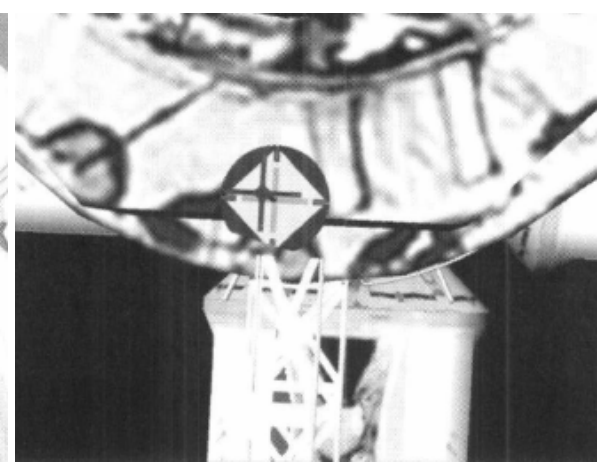

(b) Russia

Fig. 1. Drone of the spacecraft in the manual control mode [6]

The docking mechanism is to mate the spacecraft stably through gradual adjusting the relative velocity and attitude between the chasing vehicle and the object vehicle. Ultra complicated attitude control and mating systems will bear high workload on the astronauts. The principles what kind of factors affect the workload and how does this happen need investigation. Workload is a concept influenced by various variables, e.g., work requirement, time pressure, and the capability and engagement of the operator, etc [7]. Subsequently, work load affects the physiological balance of autonomic nerve system. In normal situations, both sympathetic and pneumogastric nerve system maintain a balance between each other dynamically. Under stress, sympathetic 
components dominate and result in unbalanced autonomic nerve system [8]. Electrocardiography (ECG), together with HRVs which are derived from ECG, is an easy, noninvasive, and instantaneous quantitative measure of autonomic nerve system activity $[9,10]$. A task Force of the European Society of Cardiology and the North American Society of Pacing Electrophysiology has once announced that HRV is the best quantification index to evaluate the activity of autonomic nervous system [11]. Researches have shown HRV values increase with the increasing of workload [12]. HRV has been applied in the assessment of workload in many areas, such as driving, aviation, automobile assembly etc. Several HRV parameters have been presented [13], such as mean heart rate (HR), standard deviation of the RR intervals between normal beats (SDNN), root mean square of successive differences (RMSSD) as time domain features; and very low frequency (VLF), low frequency (LF), high frequency (HF), the ratio of LF to $\mathrm{HF}$ (LF/HF), normalized LF (LFnu), and normalized HF (HFnu) components as frequency domain features [11]. The principles and devices of recording HRVs have been well established for decades, but compared to more conventional methods such as self reporting work load questionnaire, rarely work using HRVs in spaceflight studies has been reported.

This paper is organized as follows. In Section 2, we introduce the experiment. Section 3 reports the results. Some discussions are given in Section 4. Finally, Section 5 summarizes this study.

\section{Experiment}

\subsection{Participants}

Twenty two male technicians, aged between 22 and 35 years, from the China Astronaut Research and Training Center were recruited for this experiment. They all had at least a bachelor's degree and work experience related to manual spaceflight. All of them signed the consent forms. The preliminary phase was screening the participants. Each participant was tested by using DXC-6 Psychological Assessment Instrument (developed by the Fourth Military Medical University, Xi'an, China). Tests included velocity estimate test, rotation test and both-hand coordination ability test. Finally, sixteen persons who passed the tests with a threshold score were admitted to participate in the following experiment. Before the experiment began, the participants took part in the RVD theory course. They were taught the basic knowledge and scientific disciplines of RVD techniques.

\subsection{Design}

A within-participants design for operation difficulty level and between-participants design for training method is proposed in this study. Sixteen participants were divided into two sub-groups via different training method. Each participant performed an operation series twice, each series constitute of nine operation units, classified into three difficulty levels. Before the formal experiment, each participant conducted three turns of trials to get familiar with the operations. 
Two training methods, routine method and confusing method, were proposed. They are based on the astronaut training experience. Before the experiment, the subjects were trained to finish three stages of operations, i.e., elementary training, advanced training and pre-mission training. For the sub-group of routine training method, units with the same difficulty level are performed in each stage. In other words, units with difficulty level 1 , the easiest level, were asked to be performed in stage one. Units with difficulty level 2, the median difficulty level, were performed in stage two, while difficulty level 3 in stage three. In contrast, for the sub-group of confusing training method, units with different difficulty levels are performed alternatively in each stage. That is to say, each stage has the three difficulty levels of operation levels.

For the independent variable of difficulty level, it's determined by changing the original distance and attitudes of the chasing spacecraft to the object vehicle, as well as the velocity along the each axis. Subjective evaluation incorporated with the training expertise was adopted to judge the difficulty level of each operation. For example, if the original distance and attitude was set small, and the velocity was set to not so speedy, the operation would be deemed as low difficulty level. Three difficulty levels, the easiest, the moderate and the most difficult, were determined in the end.

\subsection{Apparatus and Measures}

The final RVD phase is a complicated problem. The high accuracy of relative position and attitude determination is realized by manly control. The image of the cross drone under the docking interface of the object vehicle was recorded by a television camera shooting system. Then the image was displayed onto the screen in front of the astronaut [14]. The cross drone images guide the astronauts to the object vehicle while adjusting the chasing vehicle's velocity and attitude carefully and gradually. The ideal mode of the chasing vehicle is in the zero state mode which means there is neither displacement nor attitude deviation for the chasing vehicle to the object vehicle. The cross drone may tilt, roll, yawn or displace in each axis during the RVD. The astronaut has to manually adjust the controllers to manipulate the chasing vehicle to change it into the zero state mode.

The electrocardiography of the participants was recorded by the Physiological indices recording detector at multiple time points. The detectors were worn on the participants next to the skin while performing the RVD tasks. KF2 physiological parameter analyzing software developed by a research laboratory in China was used to extract the physiological parameters from the electrocardiography data. In order to eliminate the bias resulted from the specific physiological characteristics of each participant, the original absolute physiological parameters during the RVD were substituted by new measures which were obtained by dividing their corresponding absolute values when the participants were in quiet status just before the experiment began.

\section{Results}

SPSS 16.0 was used for the data analysis in this study. All physiological parameters, i.e., the body temperature, the respiratory rate, the heart rate, the natural logarithmic values of VLF, the square root of $\mathrm{LF}$, the cubic root of $\mathrm{HF}, \mathrm{LFNu}$, the natural logarithmic values of $\mathrm{HFNu}$, and the natural logarithmic values of $\mathrm{LF} / \mathrm{HF}$, passed the 
normality test by Kolmogorov-Smirnov method, but the respiratory rate together with the natural logarithmic values of LF/HF failed the homogeneity of variances test by Levene's method. Non-parameter tests were conducted on the respiratory rate and the natural logarithmic values of $\mathrm{LF} / \mathrm{HF}$, while other physiological parameters were analyzed using two-way ANOVA. The results of the analysis are shown in Tables 1-4 and Figs. 2-3. Results show that no significant effect of the interaction was found ( $p$ values are $0.974,0.842,0.871,0.802,0.462,0.824$ and 0.856 for the body temperature, the heart rate, the natural logarithmic values of VLF, the square root of LF, the cubic root of $\mathrm{HF}, \mathrm{LFNu}$ and the natural logarithmic values of $\mathrm{HFNu}$, respectively).

Table 1. ANOVA of physiological parameters between training methods

\begin{tabular}{|c|c|c|}
\hline dependent variable & $\mathrm{F}$ value & Sig. \\
\hline body temperature & 8.00 & 0.010 \\
\hline respiratory rate ${ }^{\mathrm{a}}$ & - & 0.000 \\
\hline heart rate & 15.827 & 0.000 \\
\hline $\ln ^{\mathrm{b}}(\mathrm{VLF})$ & 0.419 & 0.518 \\
\hline square root LF & 2.957 & 0.087 \\
\hline cubic root $\mathrm{HF}$ & 4.188 & 0.042 \\
\hline $\mathrm{LFNu}$ & 6.913 & 0.009 \\
\hline $\ln ^{\mathrm{b}}(\mathrm{HFNu})$ & 2.141 & 0.145 \\
\hline $\ln ^{\mathrm{b}}(\mathrm{LF} / \mathrm{HF})^{\mathrm{a}}$ & - & 0.100 \\
\hline
\end{tabular}

a. No parameter Mann-Whitney test. ${ }^{b}$. In means the natural logarithmic value.

ANOVA for the cubic root of HF, and LFNu ( $p$ values are 0.042 and 0.009 , respectively) showed statistically significant effects of training method. Post hoc pair wise comparisons of the cubic root of HF showed that the means for routine training method were significantly higher than that for confusing training method, while comparisons of LFNu showed opposite trend, as shown in Fig.2 and Table 2. This can be explained as follows. In routine training method, the participants were trained by the operations on the same difficulty level in each stage, and each stage consists of nine operation units. The monotony in each stage made the participants feel humdrum and sterile; consequently this procedure would distract their attention from the operations and made them relaxed. However, in confusing training method, the participant was trained with all kinds of difficulty levels alternatively in each stage. This frequent change of operation difficulty led to tension of their brain pallium, made it not easy for the participants to relax themselves. They had nothing but to focus on the operations. Consequently, by using routine training method can result in lower mental workload on the operators than by using the confusing training method. These results conform with the view of Kim [8]. Their research team once announced that workload decreases high frequency components in HRV while increases low frequency components. 


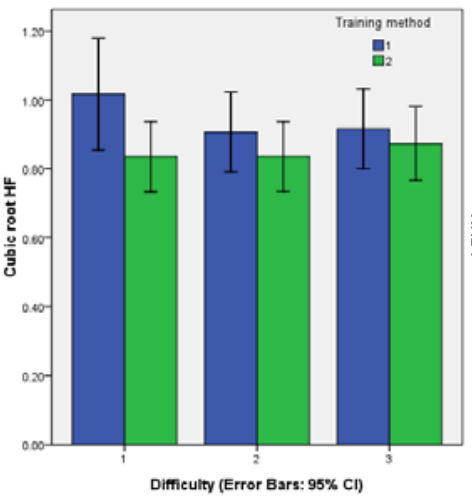

(a)

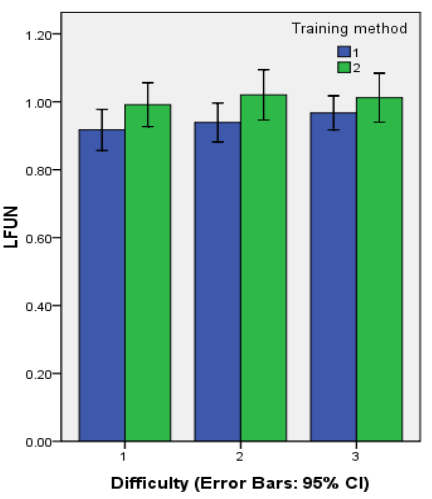

(b)

Fig. 2. Cubic root of High Frequency (HF) (a) and normalized Low Frequency (LFNu) (b) for different training methods and difficulty levels during RVD. Blue denotes routine training method, and green denotes confusing training method. Both cubic root of HF and LFNu are the ratios compared with the corresponding values when the participants are in quiet status just before the experiment began.

Table 2. Statistics of High Frequency (HF) and normalized Low Frequency (LFNu)/

\begin{tabular}{cccccc}
\hline \multirow{2}{*}{$\begin{array}{c}\text { physiological } \\
\text { parameter }\end{array}$} & difficultly & \multicolumn{4}{c}{ training method } \\
\cline { 3 - 6 } & level & \multicolumn{2}{c}{ routine method } & confusing method \\
\cline { 3 - 6 } & 1 & 1.02 & 0.45 & 0.84 & 0.28 \\
\hline \multirow{2}{*}{$\begin{array}{c}\text { cubic root of } \\
\text { HF ratio }\end{array}$} & 2 & 0.91 & 0.32 & 0.84 & 0.28 \\
& 3 & 0.92 & 0.32 & 0.87 & 0.30 \\
& 1 & 0.92 & 0.17 & 0.99 & 0.18 \\
LFUN ratio & 2 & 0.94 & 0.16 & 1.02 & 0.21 \\
& 3 & 0.97 & 0.14 & 1.01 & 0.20 \\
\hline
\end{tabular}

Results demonstrate significant training method effect on the body temperature ( $p$ value is 0.01 ). Furthermore, significant difference on the body temperature ( $p$ value is 0.01 ) was also found between different operation difficulty levels. While for the natural logarithmic values of VLF, the differences between ether the training methods or the difficulty levels were not significant. This seems incompatible with the medical common sense that VLF, component smaller than $0.04 \mathrm{~Hz}$, primarily associates with the regulation of body temperature [15]. But if we depict the comparisons of body temperature and VLF values between training methods and difficulty levels as shown in Fig. 3, it can be seen that with the increase of the difficulty level, the body temperature increases gradually. This trend also happens on VLF. Maybe the variation of workload is not big enough to 
influence VLF significantly, but both VLF and the body temperature share the same trend indeed. Statistics of body temperature and VLF were shown in Table 3.

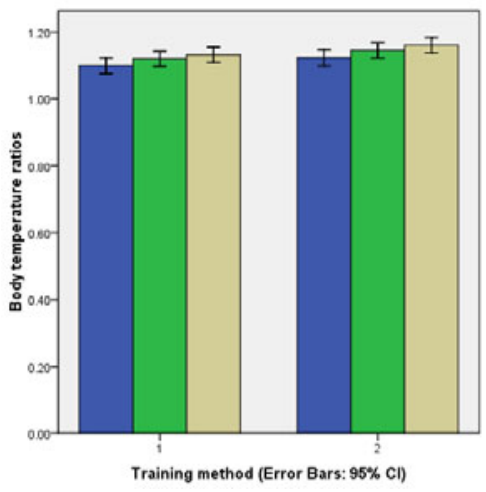

(a)

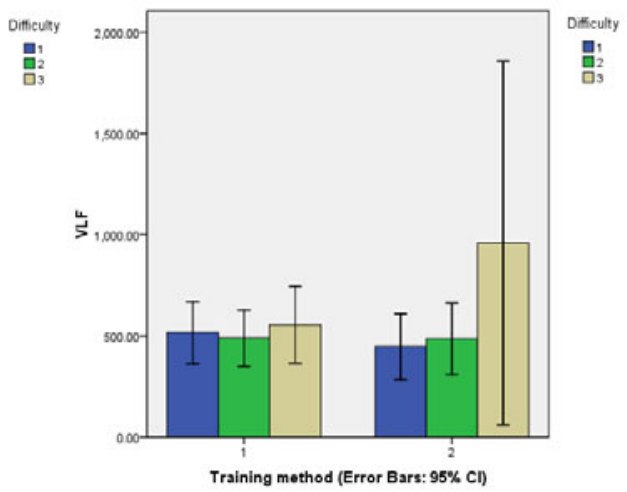

(b)

Fig. 3. Body temperature (a) and very Low Frequency (VLF) (b) for different training methods and difficulty levels during RVD

Table 3. Statistics of body temperature and very Low Frequency (VLF)

\begin{tabular}{cccccc}
\hline \multirow{2}{*}{$\begin{array}{c}\text { physiological } \\
\text { parameter }\end{array}$} & difficultly & \multicolumn{4}{c}{ training method } \\
\cline { 3 - 6 } & level & routine method & confusing method \\
\cline { 3 - 6 } & & M. & Std. & M. & Std. \\
\hline \multirow{2}{*}{ body temperature ratio } & 1 & 1.10 & 0.06 & 1.12 & 0.07 \\
& 2 & 1.12 & 0.06 & 1.15 & 0.06 \\
natural logarithmic value of & 1 & 1.13 & 0.06 & 1.16 & 0.06 \\
VLF ratio & 2 & -0.28 & 0.91 & -0.48 & 1.26 \\
& 3 & -0.34 & 1.02 & -0.43 & 1.18 \\
& & -0.21 & 0.82 & -0.22 & 1.15 \\
\hline
\end{tabular}

ANOVA for the respiratory rate and the cubic root of HF showed statistically significant effects of training method ( $p=0.000$ and 0.042 , respectively). Post hoc pair wise comparisons show the respiratory rate and HF share the similar trend between training methods. The average values of the respiratory rate and HF for the routine training method are both higher than those for the confusing training method. This result is in accordance with the explanation as follows: HF, the relatively high frequency component between $0.15-0.40 \mathrm{~Hz}$, corresponds to the frequency of respiration [15].

In the result analysis related with the task difficulty levels, no clear correlation between the difficulty of the task and HRV was found, as shown in Table 4. 
Table 4. ANOVA of physiological parameters between difficulty levels

\begin{tabular}{|c|c|c|}
\hline dependent variable & F value & Sig. \\
\hline body temperature & 4.84 & 0.010 \\
\hline respiratory rate $\mathrm{a}^{\mathrm{a}}$ & - & 0.525 \\
\hline heart rate & 0.265 & 0.768 \\
\hline $\ln ^{\mathrm{b}}(\mathrm{VLF})$ & 0.523 & 0.594 \\
\hline square root LF & 0.200 & 0.819 \\
\hline cubic root HF & 0.442 & 0.643 \\
\hline $\mathrm{LFNu}$ & 0.691 & 0.502 \\
\hline $\ln ^{\mathrm{b}}(\mathrm{HFNu})$ & 0.227 & 0.797 \\
\hline $\ln ^{\mathrm{b}}(\mathrm{LF} / \mathrm{HF})^{\mathrm{a}}$ & - & 0.623 \\
\hline
\end{tabular}

\section{Discussions}

More thorough understanding about physiological characteristics under real working situations need to be addressed from both theoretically and in practice. In fact, arguments about the relationship between physiological parameters and work load never disappear all the times. No optimal parameter has been presented so far. It's once addressed that workload decreases high frequency components in HRV while increases low frequency components [8]. But Ryu and Myung thought that an increase in mental workload was typically related to a reduction in the power associated with the mid-frequency band in the HRV, implying a temporary suppression of normal arterial pressure regulation [16]. Others argued that HRV features representing high frequency components could be more accurately estimated [17], while HRV features representing low frequency components have poor repeatability and could not be estimated accurately from ECG segments shorter than $10 \mathrm{~min}$ [18]. Researchers also pointed out that a single physiological measure may not provide adequate information to assess the workload [16].

In this experiment, no difference between operation difficulty levels was found on the majority of physiological parameters. This can be explained as follows. Before the formal experiment, each participant conducted three turns of trials. After these trials, the participants had gotten used to the operational patterns. This training effect decreases the influence of the operation difficulty on the physiological workload. Another reason may be that the difficulty level was determined empirically by just changing the distance, velocity and attitudes of the chasing vehicle to the object vehicle. It's assumed that variation of the controllers' parameters will absolutely affect the difficulty level. However, this hypothesis should be validated in advance. Furthermore, operation complexity theory could be referred to develop spaceflight operation difficulty measures to quantify the difficulty level of RVD operations [19]. An interesting thing is that Jorna concluded spectral measures are not sensitive to increased difficulty levels within the same type of task [20]. 
Some researchers argue that subjective workload evaluation approaches, such as NASA Task Load Index (NASA-TLX) questionnaire, should be also considered in the assessment of the participants' workload. Actually, typical subjective workload measures such as Cooper-Harper measure has been widely used in assessment of work load. A multivariate workload evaluation index, which integrates physiological parameters and one subjective parameter through Principal Component Analysis was proposed by Miyake [21]. However, no matter how honestly the subjects try to recall his or her feelings during the task, subjective data is not reliable or comprehensive enough [22]. The oblivion effect will get worse if the task lasts too long time. It's too difficult or even impossible to prevent some subjective bias as well. To develop a real time subjective workload assessment method is a meaningful direction (as done in the latest Observer product of Noldus Co. Ltd, www.noldus.com), whereas this is not easy since the operators have no time to conduct such kind of a questionnaire during the task, especially in the emergency environment such as firefighting, aviation and spaceflight.

\section{Conclusions}

This study is intended to serve as a starting point in developing effective and efficient training methods in the future training of China astronauts. With 16 technicians recruited, the influencing factors for operator's workload in manual controlled RVD by using physiological parameters were addressed. Results show significant effects imposed by training methods on physiological parameters. However, no significant effect of task difficulty level has been found. More subjects and experiments would be demanded for further investigation of the effectiveness of physiological parameters for the evaluation of operator's mental workload in RVD. What is more, the relationship between physiological parameters and operation performance is worth further investigation in future. Besides RVDs, the proposed method could be applied to other fields such as aviation, driving, and nuclear power plant control etc.

Acknowledgments. This research was supported by China Manned Space Project Fund 2009SY5411005 and the National Natural Science Foundation of China under grant 71001092. The authors express gratefulness to the subjects for their participation in the experiment.

\section{References}

1. Zhu, R.Z.: Rendezvous and docking techniques of spacecraft. National Defense Industry Press, Beijing (2007)

2. Zhang, Y.J., Li, Z.Z., Wu, B., Wu, S.: A spaceflight operation complexity measure and its experimental validation. International Journal of Industrial Ergonomics 39, 756-765 (2009)

3. Ui, K., Matunaga, S., Satori, S., Ishikawa, T.: Microgravity Experiments of Nano-Satellite Docking Mechanism for Final Rendezvous Approach and Docking Phase, pp. 56-63 (2005)

4. Hall, R.D., Shayler, D.J.: Soyuz: A universal spacecraft. Springer, Berlin (2006)

5. Jiang, Z.C., Zhou, J.P., Wang, Y.F.: Manual control rendezvous and docking simulation based on cross drone. National Univ. of Defense Technology 29(7), 100-103 (2007) (in Chinese) 
6. Yang, J., Jiang, G.H., Chao, J.G.: A Cross Drone Image-Based Manual Control rendezvous and docking Method. Journal of Astronautics 31(5), 1398-1404 (2010) (in Chinese)

7. Moray, N.: Mental Workload: Its Theory and Measurement, pp. 5-6. Plenum Press, New York (1979)

8. Kim, D., Seo, Y., Kim, S.H., Jung, S.: Short Term Analysis of Long Term Patterns of Heart Rate Variability in Subjects under Mental Stress. In: International Conference on BioMedical Engineering and Informatics, pp. 487-491 (2008)

9. Zhong, X., Hilton, H.J., Gates, G.J., Jelic, S., Stern, Y., Bartels, M.N., DeMeersman, R.E., Basner, R.C.: Increased sympathetic and decreased parasympathetic cardiovascular modulation in normal humans with acute sleep deprivation. J. Appl. Physiol. 98(6), 2024-2032 (2005)

10. Henelius, A., Hirvonen, K., Holm, A., Korpela, J., Muller, K.: Mental Workload Classification using Heart Rate Metrics. In: Proceedings of 31st Annual International Conference of the IEEE EMBS, Minneapolis, Minnesota, USA, September 2-6 (2009)

11. Malik, M., Bigger, J.T., Camm, A.J., et al.: Heart rate variability: standards of measurement, physiological interpretation and clinical use. European Heart Journal 17(5), 354-381 (1996)

12. Di Rienzo, M., Mancia, G., Parati, G., et al.: Blood Pressure and Heart Rate Variability-Computer Analysis, Modeling and Clinical Applications, pp. 123-127. IOS Press, Amstedam (1993)

13. Allen, J.J.B., Chambers, A.S., Towers, D.N.: The many metrics of cardiac chronotropy: a pragmatic primer and a brief comparison of metrics. Biol. Psychol. 74(2), 243-262 (2007)

14. Jiang, Z.C., Zhou, J.P., Wang, Y.F., Li, J.R.: Manual Control Rendezvous and Docking Simulation Based on Cross Drone. Journal of National University of Defense Technology 29(5), 100-103 (2007)

15. Mulder, G.: Sinus arrhythmia and mental workload. In: Moray, N. (ed.) Mental Workload: Its Theory and Measurement, pp. 327-343. Plenum, New York (1979)

16. Ryu, K., Myung, R.: Evaluation of mental workload with a combined measure based on physiological indices during a dual task of tracking and mental arithmetic. International Journal of Industrial Ergonomics 35, 991-1009 (2005)

17. Sandercock, G.R., Bromley, P.D., Brodie, D.A.: The reliability of short-term measurements of heart rate variability. International Journal of Cardiology 103(3), 238-247 (2005)

18. McNames, J., Aboy, M.: Reliability and accuracy of heart rate variability metrics versus ECG segment duration. Med. Biol. Eng. Comput. 44(9), 747-756 (2000)

19. Zhang, Y.J., Xu, Y.Z., Li, Z.Z., Li, J., Wu, S.: Influence of Monitoring Method and Control Complexity on Operator Performance in Manually Controlled Spacecraft Rendezvous and Docking. Tsinghua Science and Technology 13(5), 619-624 (2008)

20. Jorna, P.G.A.M.: Spectral analysis of heart rate and psychological state: a review of its validity as a workload index. Biological Psychology 34, 237-257 (1992)

21. Miyake, S.: Multivariate workload evaluation combining physiological and subjective measures. International Journal of Psychophysiology 40, 233-238 (2001)

22. Parker, R., Moore, D., Baillie, B.: Measurement of Rural Fire Fighter Physiological Workload and Fire Suppression Productivity, New Zealand Fire Service Commission Research Report Number 92 (2008) 